\title{
A new insight into the fabella at knee: the foetal development and evolution
}

\author{
Z.W. Jin' ${ }^{1}$, S. Shibata ${ }^{2}$, H. Abe ${ }^{3}$, Y. Jin' ${ }^{1}$, X.W. Li ${ }^{1}$, G. Murakami ${ }^{4}$ \\ ${ }^{1}$ Department of Anatomy, Histology and Embryology, Yanbian University Medical College, Yanji, China \\ 2Department of Maxillofacial Anatomy, Graduate School of Tokyo Medical and Dental University, Tokyo, Japan \\ ${ }^{3}$ Department of Anatomy, Akita University School of Medicine, Akita, Japan \\ ${ }^{4}$ Division of Internal Medicine, Iwamizawa Asuka Hospital, Iwamizawa, Japan
}

[Received: 21 June 2016; Accepted: 9 August 2016]

Using longitudinal semiserial sections of 12 lower extremities from 8 human foetuses at 15-18 weeks, we compared foetal morphologies of the knee in specimens with and without fabellae. We also compared the fabella, if present, with the hallucal sesamoid in the same foetus. Cartilaginous fabella, positive for versican and tenascin by immunohistochemistry, was found in 5 of the 8 foetuses. This structure was embedded in a thick and tight lateral fibrous band, providing a common origin of the plantaris muscle and the lateral head of the gastrocnemius muscle. The plantaris was covered by the lateral head of the gastrocnemius, but these 2 muscles were separated by a distinct fascia or space. Notably, the foetal fabella did not attach to the joint capsule. In the 3 specimens without fabellae, the lateral fibrous band was thin, containing a fibrous mass, negative for versican and tenascin, in place of the fabella. The "medial" head of the gastrocnemius faced or covered the plantaris, while the lateral head was continuous with the plantaris. A hallucal cartilaginous sesamoid, positive for versican and tenascin, was present in all 8 specimens. It carried a flat surface facing the joint cavity and was covered by tendons of the short muscles of the foot. Because of the difference in topographical relation of muscles between specimens with or without fabella, rather than mechanical stress to the tendon, fabella development may require a distinct plantaris muscle independent of the gastrocnemius. We discussed about an evolutionary aspect of the fabella and plantaris muscle. (Folia Morphol 2017; 76, 1: 87-93)

Key words: fabella, knee, hallucal sesamoid, metatarsophalangeal joint, gastrocnemius muscle, human foetus

\section{INTRODUCTION}

The fabella is a sesamoid bone present at the tendinous origin of the lateral head of the gastrocnemius muscle. The incidence and bony nature of fabellae in human adults has been found to vary, with studies showing incidence rates of $66.6 \%$ (44\% bony) [8]; $23.5 \%$ (72\% bony) [13]; $86.9 \%$ (56\% bony) [24]; and $31 \%$ (100\% bony) [3]. Almost half of fabellae carry a facet for articulation to the lateral femoral condyle [3]. A bony, but not an elastic-textured, fabella has been found to correlate with thickening of the fabellofibular ligament to strengthen the posterolateral corner of the knee [11]. The fabella has also been associated with pathologic conditions, causing pain [15] and/or osteoarthritis [14, 19]. Overall, the adult morphology of the fabella seems not to be stable. 
The sesamoid bone is generally thought to be formed by a compressive load on the tendon $[2,16]$. The establishment in foetuses of the muscle belly, including the tendinous origin of the gastrocnemius, may be followed by the appearance of the cartilaginous fabella, depending on the activities of muscles that move the knee and ankle. This developmental sequence is quite different from those of the cartilaginous femur, tibia and fibula, in that they start to develop earlier than their associated muscles, tendons and ligaments [6,9]. Moreover, in adults, the fabella is sometimes or often absent and, even if present, it often fails to contact to the knee joint (see above). In contrast, a sesamoid bone facing the joint cavity is always present at the plantar and medial aspects of the first or hallucal metatarsophalangeal joint [22, 23].

Consequently, with an aid of immunohistochemical assays of matrix proteins, we compared foetal morphologies of the knee in specimens with and without fabella. Even when the fabella is absent, the matrix may suggest an anlage undetectable with routine staining. Whether the fabella is present or absent may depend on a difference in the muscle anatomy surrounding it because mechanical loading from the muscles seems to be critically important for development. To show typical staining of matrix, we also examined the hallucal sesamoid in the same foetus.

\section{MATERIALS AND METHODS}

The study was performed in accordance with the provisions of the 1995 Declaration of Helsinki, as revised in 2013. Our previous study on posterolateral fibrous bands of the knee showed that the fabella starts to appear at gestational age 15 weeks [12]. Similarly, the hallucal sesamoid also appears to develop at 14-16 weeks [20]. Therefore, we chose midterm foetuses for the present materials.

Longitudinal semiserial sections were obtained from 12 knees and toes of 8 mid-term human foetuses of gestational age 15-18 weeks (crown-rump length, 115-155 mm), including 2 foetuses each of gestational ages 15 and 16 weeks and 4 of gestational age 18 weeks. These foetuses were parts of a collection maintained in the Department of Anatomy of Akita University, Akita, Japan. They had been donated to the Department by their families during 1975-1985, fixed by immersion in $10 \% \mathrm{v} / \mathrm{v}$ neutral formalin solution and stored in $50-70 \% \mathrm{v} / \mathrm{v}$ ethanol solution for more than 30 years. The available data were limited to the date of donation and the number of gestational weeks.
Family names, names of obstetricians or hospitals and the reasons for abortion were unavailable. Their use for research was approved by the Akita University Ethics Committee (No. 1428).

After dividing the body into parts, the lower extremities were decalcified by incubating in $0.5-\mathrm{mol} / \mathrm{L}$ EDTA ( $\mathrm{pH} 7.5$ ) solution (Decalcifying Solution B; Wako, Tokyo, Japan) at $4^{\circ} \mathrm{C}$ for 3-5 days, depending on the size of the sample. Twelve paraffin blocks, each of which contained the knee, leg and foot, were prepared from the 8 mid-term foetuses. Each block was used to prepare $80-120$ semiserial sections covering the entire knee and toe; each section was 5 microns thick with an interval between sections of 20-50 microns. Every fifth section was stained with haematoxylin and eosin (HE), while the other four sections were used for immunohistochemistry. Sections were pretreated with testicular hyaluronidase $(25 \mathrm{mg} / \mathrm{mL}$; Sigma type I-S; Sigma Chemicals, St. Louis, MO, USA) in phosphatebuffered saline for $30 \mathrm{~min}$ at $37^{\circ} \mathrm{C}$ (Shibata et al. 2003), followed by incubation with the primary antibodies, mouse monoclonal anti-versican core protein (12C5) (Developmental Studies Hybridoma Bank, diluted 1:25) and rabbit polyclonal anti-rat tenascin-c (Chemicon, Temecula, CA, USA; diluted 1:100) overnight at $4^{\circ} \mathrm{C}$. The samples were subsequently incubated with secondary antibody for $30 \mathrm{~min}$, followed by incubation for 30 min in Histofine Simple Stain Max-PO (Nichirei, Tokyo, Japan) for the diaminobenzidine (DAB) reaction with horseradish peroxidase (HRP). Sections were counterstained with haematoxylin. Samples were observed and photographed with a Nikon Eclipse 80 microscope.

Versican and tenascin-c are markers of chondrogenesis [18], as well as being present at the foetal cartilage-tendon interface under tensile stress [10]. Endochondral ossification occurs in a versican-rich matrix, while perichondral ossification occurs in tenascin-positive fibrous tissues [7]. Antibodies to these two proteins may therefore be associated with the development of fabella.

\section{RESULTS}

Differences in morphologies of muscles and associated connective tissues were observed in specimens with and without fabella. The following sections describe the morphologies of the two types of specimen.

\section{HE-stained sections of cartilaginous fabellae}

Oval, cartilaginous fabellae (maximum diameter $0.2-0.3 \mathrm{~mm}$ ) were observed in 5 of the 8 foetuses and 


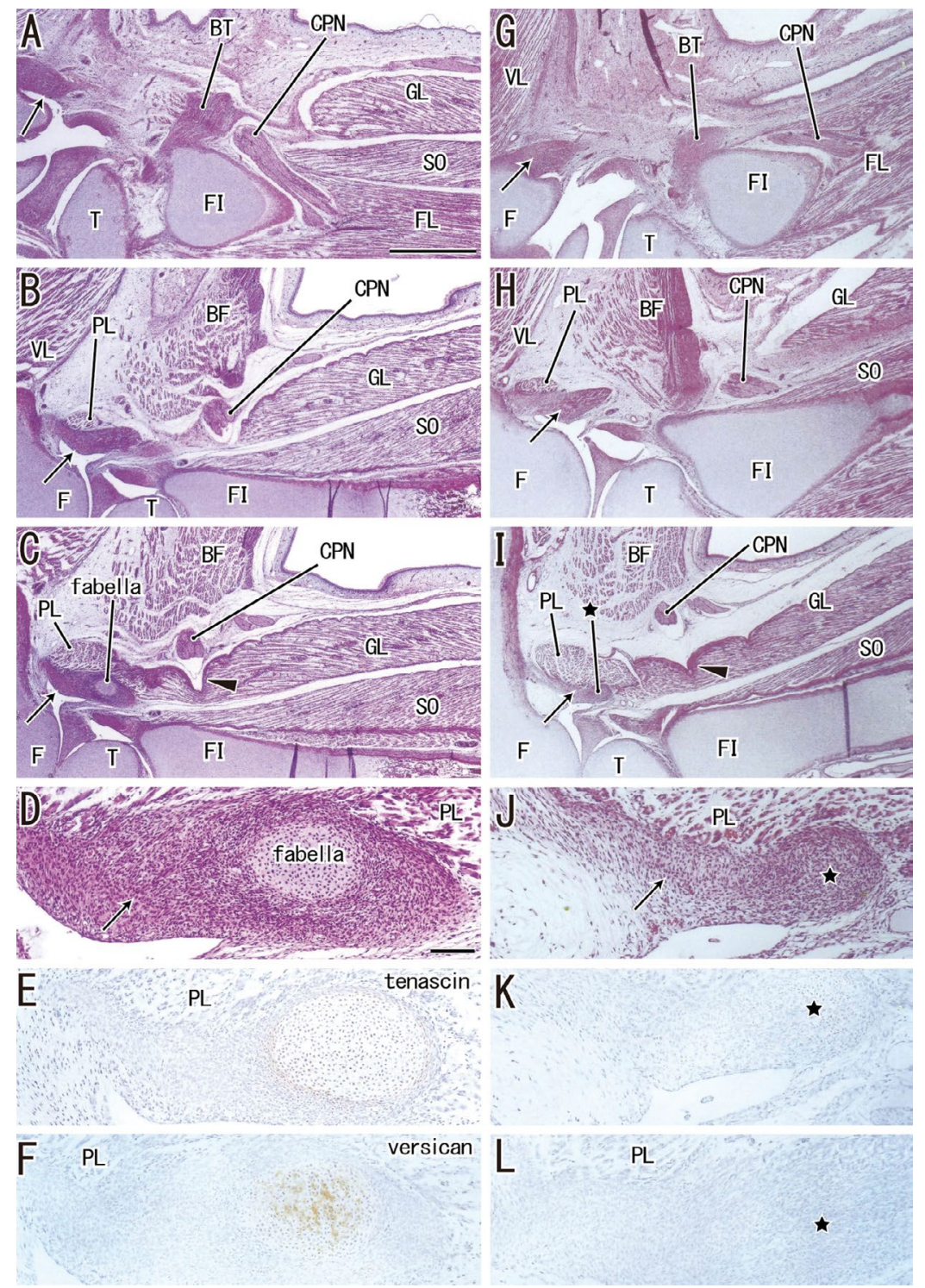

Figure 1. Plantaris and gastrocnemius muscles with or without a fabella (lateral part). The figure shows haematoxylin and eosin (HE)-stained longitudinal sections of two specimens of gestational age 15 weeks, one with $(\mathbf{A}-\mathbf{F})$ and the other without $(\mathbf{G}-\mathbf{L})$ a fabella. Panels $\mathrm{D}$ and $\mathrm{J}$ are higher magnifications view of areas in panels $\mathrm{C}$ and $\mathrm{I}$, respectively, that included the plantaris muscle. Panels $\mathrm{E}$ and $\mathrm{K}$ show immunohis tochemical staining for tenascin-c, while panels $F$ and $L$ show immunohistochemical staining for versican; with all showing sections near panels $C$ and $\mathrm{I}$, respectively. Intervals between panels are $0.7 \mathrm{~mm}(A-B)$, $0.4 \mathrm{~mm}(\mathrm{~B}-\mathrm{C}, \mathrm{G}-\mathrm{H})$ and $0.6 \mathrm{~mm}(\mathrm{H}-\mathrm{I})$. In all panels, arrows indicate the lateral fibrous band for origins of the gastrocnemius and plantaris muscles. In panels $A$ and $G$, the biceps tendon (BT) is inserted to the fibula and the common peroneal nerve (CPN) reaches the fibularis longus muscle (FL). In panels $B$ and $H$, the plantaris muscle (PL) appears along the lateral fibrous band. Panels $C$ and I show a notch (arrowhead) on the gastrocnemius lateral head (GL) for the common peroneal nerve. Panels $\mathrm{C}-\mathrm{F}$ show a cartilaginous fabella, while panels $\mathrm{I}-\mathrm{L}$ show a dense fibrous mass (star). Panels $A-C$ and $\mathrm{G}-\mathrm{I}$ were prepared at the same magnification, as were panels D-F and J-L. Scale bars: $1 \mathrm{~mm}$ in panel $A ; 0.1 \mathrm{~mm}$ in panel $D$. $\mathrm{BF}$ - biceps femoris muscle; $\mathrm{F}$ - femur; $\mathrm{FI}$ - fibula; SO — soleus muscle; $\mathrm{T}$ tibia; $\mathrm{VL}$ — vastus lateralis muscle.
9 of the 12 knees. Fabellae were present bilaterally in 4 foetuses and unilaterally in the fifth (Figs. 1, 2). Each fabella was embedded in a thick, tight and long fibrous band, called a lateral fibrous band in this study, connecting the lateral head of the gastrocnemius muscle to the lateral condyle of the femur (Figs. 1B, C; 2A). The plantaris muscle fibres were found to originate from the posterolateral aspect of the lateral fibrous band without interposition of any short tendons (Fig. 1B). The fibrous band was attached tightly to the joint capsule on the proximal side of the lateral meniscus. The lateral head of the gastrocnemius covered almost the entire posterior aspect of the plantaris muscle belly from the posterior side (Fig. 2A-C). At the inferior end, the plantaris muscle was separated from the lateral head of the gastrocnemius muscle by a distinct space or fascia (Fig. 2A-D).

The common peroneal nerve was attached to the lateral head of the gastrocnemius muscle and provided a deep notch on the muscle belly. The plantaris tendon crossed the lateral side of the tibialis nerve and extended inferiorly between the soleus and gastrocnemius muscles. The fabella was located close to the joint capsule, but was not exposed to the joint cavity. A thin ligamentous structure originated from the fabella and extended medially on the immediately posterosuperior side of the popliteus muscle (Fig. 2C-E). A similar ligament appeared to be present in specimens without fabella (see below). In addition, the origin of the medial head of the gastrocnemius 

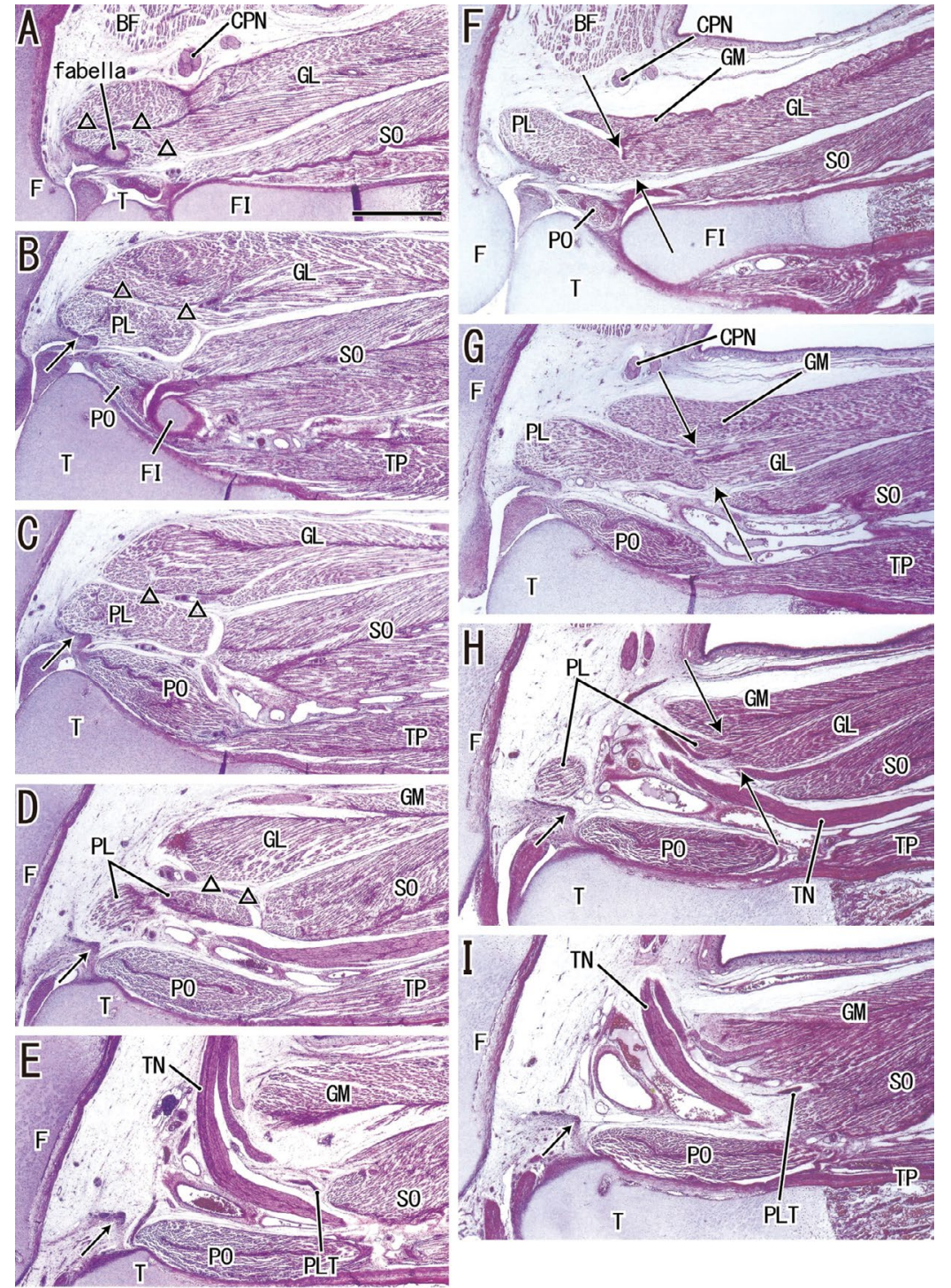

Figure 2. Plantaris and gastrocnemius muscles with or without a fabella (medial part). Continuation of Figure 1. Panel $A$ is $0.6 \mathrm{~mm}$ medial to Figure $1 \mathrm{C}$, while panel $\mathrm{F}$ is $0.4 \mathrm{~mm}$ medial to Figure 1I. Intervals between panels are $0.5 \mathrm{~mm}(A-B), 0.2 \mathrm{~mm}(B-C)$, $0.3 \mathrm{~mm}(\mathrm{C}-\mathrm{D}, \mathrm{D}-\mathrm{E}), 0.6 \mathrm{~mm}(\mathrm{~F}-\mathrm{G}), 0.4 \mathrm{~mm}$ $(\mathrm{G}-\mathrm{H})$, and $0.2 \mathrm{~mm}(\mathrm{H}-\mathrm{I})$. Panels A-E show a specimen with a fabella, while panels $\mathbf{F}-\mathbf{I}$ show a specimen without a fabella. In all panels, short arrows indicate a thin ligamentous structure originating from the fabella or fibrous mass and extending medially. Panels A-D show the gastrocnemius lateral head (GL) covering the plantaris muscle (PL), with the lateral head separated from the plantaris by a fascia or space (triangles). Panels $\mathrm{F}-\mathrm{H}$ show the medial head (GM) facing or covering the plantaris, with the lateral head and plantaris muscles continuous at a site sandwiched by long arrows in panels $\mathrm{F}-\mathrm{H}$. Panels $\mathrm{E}$ and I show the plantaris tendon (PLT) and tibial nerve (TN). All panels were prepared at the same magnification (scale bar: $1 \mathrm{~mm}$ in panel A); BF — biceps femoris muscle; $\mathrm{CPN}$ - common peroneal nerve; $\mathrm{F}$ - femur; $\mathrm{FI}$ - fibula; $\mathrm{PO}$ - popliteus muscle; $\mathrm{SO}$ - soleus muscle; T — tibia; $\mathrm{TP}$ - tibialis posterior muscle. muscle was not tendinous but composed of a small mass of muscle fibres.

\section{HE-stained sections without fabellae}

The remaining 3 knees of 3 foetuses lacked fabella; in these knees, the lateral fibrous band was thin but present (Fig. 1). The band contained a fibrous mass instead of a fabella (Fig. 1I) and was attached to the joint capsule on the proximal side of the lateral meniscus. The lateral fibrous band remained as a thick fascia along the anterior aspect of the plantaris muscle (Fig. 2) and continued to a thin ligamentous structure running medially (Fig. $2 \mathrm{H}, \mathrm{I})$. The origin of the plantaris muscle had the similar morphology as in specimens with fabellae. Notably, the "medial" head of the gastrocnemius muscle in specimens without fabella faced or covered the plantaris muscle from the posterior aspect (Fig. 2G, H). Moreover, at the inferior end, the plantaris muscle was continuous with the gastrocnemius lateral head (Figs. 11, 2F, G). Therefore, the gastrocnemius medial head in these samples appeared to extend more laterally than specimens with fabella, while the lateral head and plantaris provided a single slender muscle belly. As in specimens with fabella, the plantaris tendon crossed the lateral side of the tibialis nerve and extended inferiorly. A deep notch by the common peroneal nerve was seen on the muscle belly of the gastrocnemius.

\section{HE-stained sections that included the hallucal sesamoid}

The cartilaginous hallucal sesamoid was present in all specimens (Fig. 3). Each had a flat dorsal surface facing the joint cavity as well as an oval shape on the plantar 

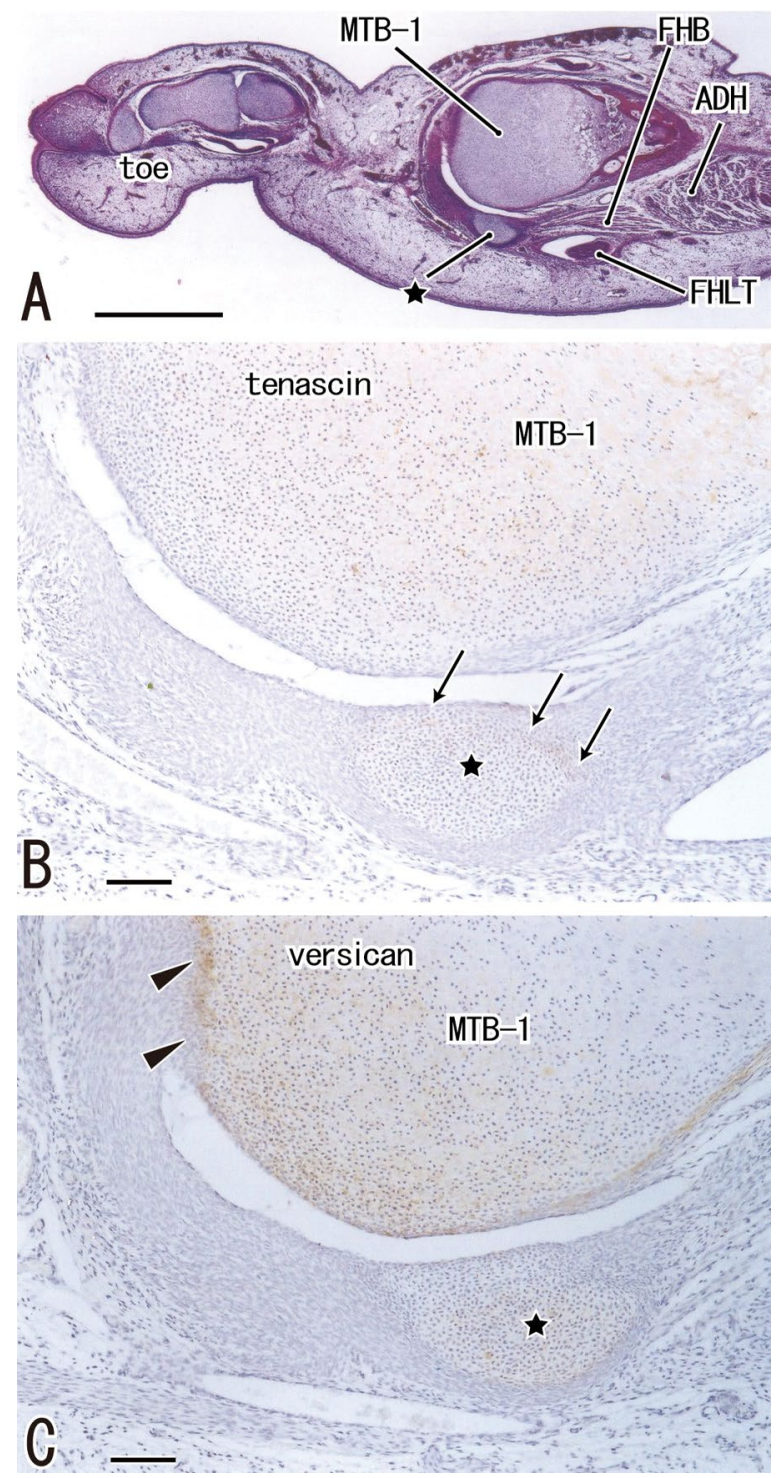

Figure 3. Foetal sesamoid at the first metatarsophalangeal joint. A toe from the 15 week old specimen illustrated in Figure 1. Panels A-F, and Figure 2, Panels A-E, was sectioned longitudinally. Panels B and $\mathbf{C}$, which show immunohistochemical staining with antibodies to tenascin-c and versican, respectively, are higher magnification views of the first metatarsal bone (MTB-1) and sesamoid (star) shown in panel $\mathbf{A}$ (HE staining). Panel $\mathbf{A}$ shows the insertion of the flexor halluces brevis muscle (FHB) into the sesamoid; insertion of the adductor halluces muscle (ADH) into the sesamoid was observed in sections $0.3 \mathrm{~mm}$ lateral to panel $A$. Tenascin-c tended to be positive in a joint surface of the sesamoid (arrows in panel B), while versican was positive in a tendon insertion (arrowheads in panel C). Scale bars: $1 \mathrm{~mm}$ in panel A; $0.1 \mathrm{~mm}$ in panels $B$ and $C$; FHLT — tendon of the flexor halluces longus muscle.

side. A tight fibrous band, or primitive plantar collateral ligament, was found to originate from the oval cartilage mass and to be attached to the base of the proximal phalanx or to the head of the first metatarsal. Tendons of the adductor halluces and flexor halluces brevis muscles were connected to the sesamoid cartilage. The thick tendon of the flexor halluces longus muscle was located close to the plantar side of the sesamoid cartilage.

\section{Immunohistochemistry of the fabella and hallucal sesamoid}

Immunoreactivity of antibodies to versican and tenascin-c was generally weak. In the knee, the developing fabella was positive for both tenascin-c and versican (Fig. 1E, F). However, the foetal lateral fibrous band in specimens without fabella was negative for both (Fig. $1 \mathrm{~K}, \mathrm{~L}$ ). In the foot, versican reactivity was seen in the tendon insertion of the adductor halluces and flexor halluces brevis muscles into the first metatarsal bone, while the joint surface of the sesamoid was positive for tenascin-c (Fig. 3B, C).

\section{DISCUSSION AND CONCLUSIONS}

The foetal fabella developed in a thick fibrous tissue attaching tightly to the joint capsule, i.e., the lateral fibrous band in this study. Because the plantaris muscle originated from the lateral fibrous band, the fabella appeared to be embedded in the plantaris muscle origin rather than that of the lateral head of the gastrocnemius. Actually, in midterm foetuses, the plantaris muscle was almost thick as the lateral head. However, the lateral fibrous band could connect between the gastrocnemius lateral head and the lateral condyle of the femur. Thus, depending on much increased thickness and length of the lateral head in contrast to the plantaris possibly keeping its small size, the lateral fibrous band seemed to be later involved in the gastrocnemius origin. In other words, the lateral head origin would steal the fabella from the plantaris origin to provide a facet for acceptance of a mechanical stress from the strong lateral head. In this context, a question arose: do some animals carry a fabella embedded in the plantaris origin in adults?

Seebacher et al. [17] hypothesized that evolutionary development of the fabella and fabellofibular ligament occurs in combination with a change from quadrupedal to bipedal posture. Compared to a quadrupedal posture, a bipedal posture requires more stability and less rotation of the knee. Human knees, while more stable, are therefore less flexible than those of quadrupeds. The fabella and fabellofibular ligament are found to be best developed in the kangaroo, in that jumping requires great stability during forceful extension of the knee [14]. In kangaroos, the well-developed plantaris muscle originates from the fabella and provides 
superficial tendons for fingers, which play a role corresponding to the flexor digitorum superficialis in the forearm [21]. Since the plantaris muscle in the lower extremities of humans may be undergoing a process of retrogression, this muscle has often been compared with the palmaris longus muscle in the forearm [5]. However, the comparative anatomy has regarded the plantaris as a finger flexor.

This study showed that foetal topographical anatomy of the plantaris and two heads of the gastrocnemius muscle differed in foetal specimens with and without cartilaginous fabellae. Development of a cartilaginous fabella was observed when the gastrocnemius lateral head and plantaris were completely separated, but not when the two muscles formed a single belly. In the latter situation, the gastrocnemius medial head became thicker and extended more laterally than the slender plantaris-lateral head complex. As the lateral fibrous band without fabella was negative for both tenascin-c and versican (i.e., no anlage), it seemed to be unable to develop cartilage. In general, development of sesamoids is mediated epigenetically by local mechanical forces associated with skeletal geometry, posture, and muscular activity [16]. However, development of the fabella, in combination with a distinct plantaris as an independent flexor muscle, may be genetically determined. In contrast to the human fabella and plantaris, which seems to be in regression, animals with a long, thick and independent plantaris are likely to carry a distinct fabella with facet. Conversely, a human fabella with facet provides a good adaptation to the gastrocnemius much stronger than the plantaris. Human evolution may divert the fabella from the plantaris to the gastrocnemius.

Fibrocartilage is found most often within tendons that wrap around bony pulleys, as well as on the surface of the pulley in contact with the tendon [1]. A fibrocartilage enthesis, a junction zone at the attachment of tendons to long bones, serves to protect tendons against wear and tear, with gradations in microstructure observed in bone, metaplastic fibrocartilage, and tendon fibrocartilage [2]. This basic concept of an enthesis was applicable to the hallucal sesamoid. However, in spite of matrix expressions similar to the hallucal sesamoid, foetal fabellae did not face to the joint cavity in the present specimens. Why did they show no contact? In adults, almost half of fabellae do not carry a facet for articulation to the lateral femoral condyle [3]. Thus, also in foetuses, there were likely to be two types of fabellae: 1) a real sesamoid facing the joint cavity and 2) a false sesamoid without facet. Indeed, because of lim-

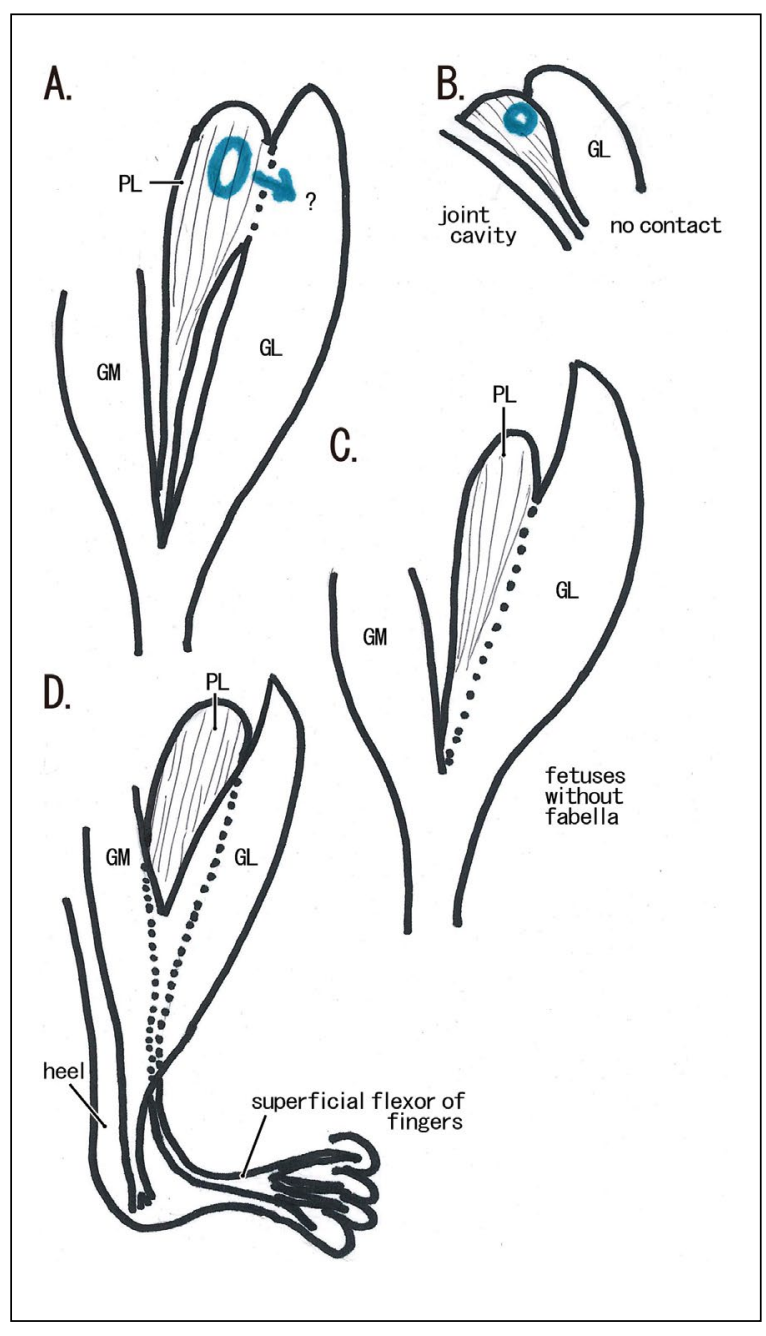

Figure 4. A summary of the present results and discussion. A close topographical relation between the fabella (blue colour) and plantaris muscle (PL) is emphasized in this figure. The fabella develops in the anterior side of the plantaris muscle when the muscle was clearly separated from the lateral head of the gastrocnemius (GL, panel A). However, the initial fabella does not face to the joint cavity (B). The fabella may be later involved into the gastrocnemius origin $(\mathbf{A}$, arrow). Foetuses with the plantaris continuous with the lateral head does not contain the fabella (C). In comparative anatomy, rather than the gastrocnemius, the fabella is associated with a well-developed plantaris acting foot fingers (D); GM — gastrocnemius medial head.

ited numbers of specimens, we might incidentally find the second type only. However, Eyal et al. [4] reported a real migration of the sesamoid: in mouse embryos, the patella originates from the femur and secondarily migrates into the quadriceps tendon. Therefore, we did not deny a possibility that, during the later development in which we hypothesized an involvement of the lateral fibrous band into the gastrocnemius origin (see the first paragraph of the 'Discussion'), the fabella could move to the joint cavity. The present results and discussions are schematically shown in Figure 4. 


\section{Acknowledgements}

This work was supported in part by a Grant-in-Aid for Scientific Research (JSPS KAKENHI No. 16K08435) from the Ministry of Education, Culture, Sports, Science and Technology in Japan.

\section{REFERENCES}

1. Benjamin $M$, Qin S, Ralphs JR. Fibrocartilage associated with human tendons and their pulleys. J Anat. 1995; 187 (Pt 3): 625-633, indexed in Pubmed:8586561.

2. Benjamin M, Ralphs JR. Fibrocartilage in tendons and ligaments--an adaptation to compressive load. J Anat. 1998; 193 (Pt 4): 481-494, doi:10.1046/j.14697580.1998.19340481.x, indexed in Pubmed: 10029181.

3. Chew CP, Lee KH, Koh JS, et al. Incidence and radiological characteristics of fabellae in an Asian population. Singapore Med J. 2014; 55(4): 198-201, doi: 10.11622/ smedj.2014052, indexed in Pubmed: 24763835.

4. Eyal S, Blitz E, Shwartz Y, et al. On the development of the patella. Development. 2015; 142(10): 1831-1839, doi: 10.1242/dev.121970, indexed in Pubmed: 25926361.

5. Frohse F, Fränkel M. Die Muskeln des menschlichen Beines. In: von Bardeleben K (ed). Handbuch der Anatomie des Menschen. Gustav Fischer, Jena, 1913; pp. 561-564.

6. Gray DJ, Gardner E. Prenatal development of the human knee and superior tibiofibular joints. Am J Anat. 1950; 86(2): 235-287, doi:10.1002/aja.1000860204, indexed in Pubmed: 15410671.

7. Hayashi S, Kim JH, Hwang SE, et al. Interface between intramembranous and endochondral ossification in human foetuses. Folia Morphol. 2014; 73(2): 199-205, doi: 10.5603/ FM.2014.0029, indexed in Pubmed: 24902099.

8. Kawashima T, Takeishi H, Yoshitomi S, et al. Anatomical study of the fabella, fabellar complex and its clinical implications. Surg Radiol Anat. 2007; 29(8): 611-616, doi: 10.1007/ s00276-007-0259-4, indexed in Pubmed: 17882346.

9. Mérida-Velasco J, Sánchez-Montesinos I, Espín-Ferra J, et al. Development of the human knee joint. Anat Rec. 1997; 248(2): 269-278, doi:10.1002/(sici)10970185(199706)248:2<269::aid-ar14>3.0.co;2-n.

10. Milz S, Benjamin M, Putz R. Molecular parameters indicating adaptation to mechanical stress in fibrous connective tissue. Adv Anat Embryol Cell Biol. 2005; 178: 1-71, doi: 10.1007/3540-27832-x, indexed in Pubmed: 16080262.

11. Minowa T, Murakami G, Kura H, et al. Does the fabella contribute to the reinforcement of the posterolateral corner of the knee by inducing the development of associated ligaments? J Orthop Sci. 2004; 9(1): 59-65, doi: 10.1007/ s00776-003-0739-2, indexed in Pubmed: 14767706.

12. Nakamura T, Suzuki D, Murakami G, et al. Human fetal anatomy of the posterior semimembranosus complex at the knee with special reference to the gastrocnemiosemimembranosus bursa. Knee. 2011; 18(4): 271-277, doi: 10.1016/j.knee.2010.05.010, indexed in Pubmed: 20797867.

13. Phukubye $P$, Oyedele $O$. The incidence and structure of the fabella in a South African cadaver sample. Clin Anat. 2011; 24(1): 84-90, doi:10.1002/ca.21049, indexed in Pubmed: 20830786.

14. Pritchett JW. The incidence of fabellae in osteoarthrosis of the knee. J Bone Joint Surg Am. 1984; 66(9): 1379-1380, doi: 10.2106/00004623-198466090-00009, indexed in Pubmed: 6501334.

15. Robertson A, Jones SCE, Paes $R$, et al. The fabella: a forgotten source of knee pain? Knee. 2004; 11(3): 243-245, doi: 10.1016/S0968-0160(03)00103-0, indexed in Pubmed: 15194103.

16. Sarin VK, Erickson GM, Giori NJ, et al. Coincident development of sesamoid bones and clues to their evolution. Anat Rec. 1999; 257(5): 174-180, doi:10.1002/(sici)10970185(19991015)257:5<174::aid-ar6>3.0.co;2-o, indexed in Pubmed: 10597342.

17. Seebacher JR, Inglis AE, Marshall JL, et al. The structure of the posterolateral aspect of the knee. J Bone Joint Surg Am. 1982; 64(4): 536-541, doi:10.2106/00004623198264040-00008, indexed in Pubmed: 7068696.

18. Shibata S, Fukada K, Imai H, et al. In situ hybridization and immunohistochemistry of versican, aggrecan and link protein, and histochemistry of hyaluronan in the developing mouse limb bud cartilage. J Anat. 2003; 203(4): 425-432, doi: 10.1046/j.1469-7580.2003.00226.x, indexed in Pubmed:14620382.

19. Theodorou SJ, Theodorou DJ, Resnick D. Painful stress fractures of the fabella in patients with total knee arthroplasty. AJR Am J Roentgenol. 2005; 185(5): 1141-1144, doi: 10.2214/AJR.04.1230, indexed in Pubmed: 16247123.

20. Uchiyama E, Kim JiH, Abe H, et al. Fetal development of ligaments around the tarsal bones with special reference to contribution of muscles. Clin Anat. 2014; 27(3): 389-398, doi: 10.1002/ca.22247, indexed in Pubmed: 23712742.

21. Warburton N, Yakovleff M, Malric A. Anatomical adaptations of the hind limb musculature of tree-kangaroos for arboreal locomotion (Marsupialia : Macropodinae). Austr J Zool. 2012; 60(4): 246, doi: 10.1071/zo12059.

22. Williams PL. Gray's Anatomy. 38th ed., Churchill Livingstone, London, 1995.

23. Yammine $K$. The sesamoids of the feet in humans: a systematic review and meta-analysis. Anat Sci Int. 2015; 90(3): 144-160, doi: 10.1007/s12565-014-0239-9, indexed in Pubmed: 24801385.

24. Zeng SX, Dong XL, Dang RS, et al. Anatomic study of fabella and its surrounding structures in a Chinese population. Surg Radiol Anat. 2012; 34(1): 65-71, doi: 10.1007/ s00276-011-0828-4, indexed in Pubmed: 21626275. 